\title{
MEETING CALENDAR 1990
}

\section{SPIE MEDICAL IMAGING IV}

February 4-9, 1990

Newport Beach, CA

For further information, contact: Society of Photo-Optical Instrumentation Engineers (SPIE), PO Box 10, Bellingham, WA 98227. Telephone, (206) 676-3290; fax, (206) 647-1445.

\section{EUROPACS 1990-PICTURE ARCHIVING AND COMMUNICATION SYSTEMS IN EUROPE}

May 3-4, 1990

Trieste, Italy

For further information, contact: Dr Paolo Giribona, CeHAB-Research Area of Trieste, Padriciano 99, 34012, Trieste, Italy. Telephone, (39) 40-226662; fax, (39) 40-226698.

S/CAR 90-SYMPOSIUM FOR COMPUTER ASSISTED RADIOLOGY

(Tenth Conference on Computer Applications in Radiology)

(Fourth Conference on Computer Assisted Radiology)

June 13-16, 1990

Anaheim, CA

For further information, contact: Ms Janice Ford, Manager, Continuing Education, Hospital of the University of Pennsylvania, 3400 Spruce St, Philadelphia, PA 19104. Telephone, (215) 662-6904.

\section{THREE-DIMENSIONAL IMAGING IN MEDICINE NATO ADVANCED RESEARCH WORKSHOP \\ June 25-29, 1990 \\ Travenmuende, Federal Republic of Germany}

For further information, contact: Ms Linda Houseman, Dept of Computer Science, Box 3175, Sitterson Hall, University of North Carolina, Chapel Hill, NC 27599. Telephone, (919) 962-1758; fax, (919) 962-1799.

MIE 90-NINTH INTERNATIONAL CONGRESS MEDICAL INFORMATICS EUROPE August 20-24, 1990

Glasgow, Scotland

For further information, contact: Dr J. S. Bryden, Information Services Unit, Royal Beatson Memorial Hospital, 132 Hill Street, Glasgow, G11 5NT, Scotland. Telephone, (44) 41-332-25 (ext 331); fax, (44) 41-332-2932. 
MEETING CALENDAR 1990 (cont'd)

VISUAL COMMUNICATIONS AND IMAGE PROCESSING '90

October 2-4, 1990

Lausanne, Switzerland

Extended summary due date: February 5, 1990

Manuscript due date: July 9, 1990

For further information, contact: SPIE Technical Program Committee/Lausanne '90, PO Box 10, Bellingham, WA 98227-0010. Telephone, (206) 676-3290; fax, (206) 647-1445.

THE SIXTH INTERNATIONAL SYMPOSIUM ON PLANNING OF

RADIOLOGICAL DEPARTMENTS (ISPRAD VI)

and

INTERNATIONAL MEETING OF HEALTH ECONOMY (IMHE)

For further information, contact: PLUSreiser A/S, PO Box 946, N-5001 Bergen, Norway. Telephone, (47) 5-314500; fax, (47) 5-324376. 